\title{
Produção científica sobre saúde do trabalhador de enfermagem em tempos de pandemia: revisão integrativa de literatura
}

Scientific production on nursing workers' health in times of pandemic: an integrative literature review

\section{Producción científica sobre la salud de los trabajadores de enfermería en tiempos de pandemia: una} revisión integradora de la literatura

\author{
Jorge Luiz Lima da Silva ${ }^{*}$, Mariana Cristine Chagas Gomes ${ }^{2}$, Fabiane Wendling Carlos ${ }^{3}$, Larissa \\ Murta Abreu $^{4}$, Larissa Vieira Correa ${ }^{5}$
}

Como citar esse artigo. Lima da Silva, JL; Gomes, MCC; Carlos, FW; Abreu, LM; Correa, LV. Produção científica sobre saúde do trabalhador de enfermagem em tempos de pandemia: revisão integrativa de literatura. Revista Pró-UniverSUS. 2021 Jan./Jun.; 12 (1): $37-46$

\section{Resumo}

Introdução: a sociedade enfrenta, atualmente, a disseminação global de um determinado vírus identificado pela primeira vez na província chinesa de Wuhan. Foi declarado estado de pandemia devido à covid-19, causada pelo novo coronavírus. Materiais e métodos: trata-se de uma revisão integrativa da literatura, utilizando-se de fontes bibliográficas secundárias, por meio de levantamento em ambiente virtual. Foi utilizado para a busca nas bases eletrônicas os descritores "saúde do trabalhador", "covid-19", "enfermagem", combinados com operador booleano "and". Resultados: a seleção foi composta por 16 artigos. No que se refere ao ano de publicação, constatou-se que, em sua totalidade (16), os artigos foram publicados no ano de 2020 mediante às publicações sobre síndrome gripal. Em sua maioria (4), os manuscritos foram publicados nos periódicos Lilacs, Medline e Scielo. Discussão: após análise textual e observação dos temas, emergiram 2 categorias levantadas a partir dos resultados encontrados, sendo: aspectos epidemiológicos relacionados à covid-19 e aspectos relacionados às condições de trabalho. Considerações finais: a partir da literatura selecionada pode-se perceber que dada a natureza recente da temática variados estudos que contemplam o tema ainda estão sendo desenvolvidos e publicados. Espera-se que a produção científica sobre a saúde dos trabalhadores de enfermagem seja fomentada durante a pandemia, pois sua contribuição é tamanha para o desenvolvimento de estratégias de prevenção de doenças e gerenciamento das emoções, revelando-se importantes componentes do enfrentamento dos profissionais a situações delicadas como a pandemia do novo coronavírus.

Palavras-chave: Saúde dos Trabalhadores; Enfermagem; Infecções por Coronavírus; Atenção à Saúde do Trabalhador

\begin{abstract}
Introduction: the society is currently facing the global spread of a virus first identified in the Chinese province of Wuhan. Pandemic status has been declared due to covid-19, caused by the new coronavirus. Materials e Methods: it is an integrative literature review, using secondary bibliographic sources, through a survey in a virtual environment. The descriptors "worker health", "covid-19", "nursing" were used to search the databases, combined with the Boolean operator "and". Results: the selection consisted of 16 articles. With regard to the year of publication, it was found that, in their entirety (16), the articles were published in the year 2020 due to the current emergence of the covid-19 pandemic. Most of them (4), the manuscripts were published in the LILACS, MEDLINE and SciELO journals. Discussion: After textual analysis and observation of the themes, 2 categories emerged from the results found, namely: epidemiological aspects related to covid-19 and aspects related to working conditions. Final considerations: From the selected literature, it can be seen that, given the recent nature of the theme, many studies that contemplate the theme are still being developed and published. It is expected that scientific production on the health of nursing workers will be promoted during the pandemic, as their contribution is so great for the development of strategies for disease prevention and management of emotions, proving to be important.
\end{abstract}

Keywords: Workers' health; Nursing; Coronavirus Infections; Attention to Worker Health.

${ }^{1}$ Docente, Programa de Pós-graduação em Saúde Coletiva/Universidade Federal Fluminense/Niterói/Rio de Janeiro/Brasil. ORCID: http://orcid.org/0000-0002-2370-6343.

${ }^{2}$ Discente, Escola de Enfermagem Aurora de Afonso Costa/Universidade Federal Fluminense/Niterói/Rio de Janeiro/Brasil. ORCID: https://orcid.org/0000-0001-8959-2054

${ }^{3}$ Discente, Escola de Enfermagem Aurora de Afonso Costa/Universidade Federal Fluminense/Niterói/Rio de Janeiro/Brasil. ORCID: https://orcid.org/0000-0002-8227-4069.

${ }^{4}$ Discente, Escola de Enfermagem Aurora de Afonso Costa/Universidade Federal Fluminense/Niterói/Rio de Janeiro/Brasil. ORCID: https://orcid.org/0000-0002-7119-4370.

${ }_{5}^{5}$ Discente, Escola de Enfermagem Aurora de Afonso Costa/Universidade Federal Fluminense/Niterói/Rio de Janeiro/Brasil. ORCID: https://orcid.org/0000-0002-3593-0131. 


\section{Resumen}

Introducción: la sociedad se enfrenta actualmente a la propagación global de un virus identificado por primera vez en la provincia china de Wuhan. Se declaró estado pandémico debido al covid-19, causado por el nuevo coronavirus. Materiales y métodos: se trata de una revisión integradora de la literatura, utilizando fuentes bibliográficas secundarias, a través de una encuesta en un entorno virtual. Para la búsqueda en las bases de datos se utilizaron los descriptores "salud del trabajador", "covid-19", "enfermería", combinados con el operador booleano "y". Resultados: la selección estuvo compuesta por 16 artículos. Con respecto al año de publicación, se encontró que, en su totalidad (16), los artículos fueron publicados en el año 2020 debido al actual surgimiento de la pandemia covid-19. La mayoría de ellos (4), los manuscritos fueron publicados en las revistas LILACS, MEDLINE y SciELO. Discusión: luego del análisis textual y la observación de los temas, de los resultados encontrados surgieron dos categorías, a saber: aspectos epidemiológicos relacionados con el covid-19 y aspectos relacionados con las condiciones laborales. Consideraciones finales: a partir de la literatura seleccionada se puede observar que, dada la naturaleza reciente del tema, aún se están desarrollando y publicando muchos estudios que contemplan el tema. Se espera que durante la pandemia se promueva la producción científica sobre la salud de los trabajadores de enfermería, pues su aporte es tan grande para el desarrollo de estrategias de prevención de enfermedades y manejo de emociones, revelándose como componentes importantes del afrontamiento de los profesionales ante situaciones delicadas. como la nueva pandemia de coronavirus.

Palabras clave: Salud de los Trabajadores; Enfermería; Infecciones por Coronavirus; Atención a la Salud del trabajador.

\section{Introdução}

Atualmente a sociedade enfrenta a disseminação global do vírusidentificado pela primeira veznaprovíncia chinesa de Wuhan. Foi declarado estado de pandemia devido à covid-19, causada pelo novo coronavírus da síndrome respiratória aguda severa (SARS-CoV-2). A doença foi iniciada em dezembro de 2019 e tem causado importantes repercussões geopolíticas, aos diversos sistemas de saúde e à economia mundial, uma vez que se dispersou rapidamente ${ }^{1}$. A Organização Mundial da Saúde (OMS) declarou estado pandêmico, o que representa emergência de saúde pública em escala mundial ${ }^{2}$.

As pandemias tendem a provocar pânico generalizado na população, principalmente quando o conhecimento sobre a doença ainda se encontra em construção. A medida mais adotada mundialmente para o controle da transmissão é o isolamento social ${ }^{3}$.

O isolamento pode intensificar os sentimentos de desamparo, tédio, solidão, tristeza e reações comportamentais como alterações ou distúrbios de apetite, distúrbios do sono e conflitos interpessoais ${ }^{3}$. Tais orientações, relacionadas ao convívio em sociedade, levaram à adoção de um novo comportamento por parte da população, causando aumento de sintomas de medo, estresse, ansiedade e depressão ${ }^{4}$.

Além do destaque pela vigência da pandemia, o ano de 2020 foi declarado pela OMS como o ano internacional da enfermagem, alertando que o mundo precisa de mais nove milhões de enfermeiros e parteiras, para atingir a meta de cobertura universal de saúde até $2030^{5}$.

$\mathrm{O}$ apontamento da necessidade dos profissionais de enfermagem emerge como demarcação da crise enfrentada anteriormente na prestação do cuidado, devido ao envelhecimento da população, cortes de verbas em serviços públicos, mudanças climáticas, dentre outros determinantes e condicionantes de saúde ${ }^{6}$.

A partir desse cenário, é válido pensar nas questões da saúde do trabalhador, entendendo que a mesma pode ser definida como campo de práticas e saberes estratégicos, interdisciplinares, multiprofissionais e interinstitucionais, que visam analisar e intervir em relações de trabalho que possam provocar doenças e agravos ${ }^{7}$.

Sabe-se que os enfermeiros enfrentam diariamente condições de trabalho instáveis, em ambientes marcados pela falta de segurança, infraestrutura inadequada e pelos riscos, que acarretam graus elevados de desgaste profissional, adoecimento físico e psicológico, má qualidade de vida e assistência à saúde.

O desgaste profissional é mais comum nas situações em que os profissionais estão diretamente em contato com pacientes infectados pelo SARS-CoV-2 e que enfrentam, além das longas horas de trabalho, preocupação com o estado de saúde dos pacientes e escassez de equipamentos de proteção individual (EPI), especialmente, em países em desenvolvimento ${ }^{8}$.

Em julho de 2020, as estatísticas do Instituto Nacional de Saúde e os diagnósticos das organizações de profissionais indicaram aumento dos casos de trabalhadores da área da saúde infectados pela covid19, sendo os enfermeiros e auxiliares de enfermagem os mais afetados por serem profissionais que atuam na linha de frente no cuidado de pessoas infectadas?.

Mediante o exposto, este estudo traz como questão de pesquisa: o que a literatura aponta sobre as possíveis consequências da pandemia à saúde do trabalhador de enfermagem? O objetivo visa descrever os impactos à saúde do trabalhador de enfermagem, no período pandêmico atual.

\section{Materiais e Métodos}

Trata-se de revisão integrativa da literatura, utilizando-se de fontes bibliográficas secundárias, por meio de levantamento de obras do ano de 2019 a 2021, em ambiente virtual. A revisão integrativa, é a mais ampla abordagem metodológica referente às 
revisões, permitindo a inclusão de variados estudos para a compreensão completa do fenômeno. Incorpora um vasto leque de propósitos: definição de conceitos; revisão de teorias e evidências; e análise de problemas metodológicos ${ }^{10}$.

Foi realizado levantamento nas seguintes bases eletrônicas: BVS (Biblioteca Virtual em Saúde); Lilacs (Literatura Latino-Americana e do Caribe em Ciências da Saúde); Scielo (Scientific Electronic Library Online), Medline (Medical Literature Analysis and Retrieval System Online) e NBCI (National Center for Biotechnology Information). Com o uso dos seguintes descritores para o carregamento das obras: "saúde do trabalhador"; "covid-19"; "enfermagem", combinados com operador booleano "and".

Os critérios de inclusão utilizados para a seleção dos artigos foram: artigos publicados nos idiomas inglês, português e/ou espanhol; texto abordando a temática da saúde do trabalhador de enfermagem durante a pandemia; indexados nas bases eletrônicas entre 2019 e 2021. Como critérios de exclusão: as obras sem relação com a temática da situação dos trabalhadores de enfermagem na pandemia.

Tanto análise, quanto a síntese dos dados extraídos dos artigos, foram realizadas de forma descritiva, possibilitando observar, contar, descrever e classificar os dados, com o intuito de reunir o conhecimento produzido sobre o tema explorado na revisão ${ }^{10}$

\section{Resultados}

Posteriormente, após a busca com auxílio dos descritores e filtragem nas bases eletrônicas, os artigos foram selecionados por meio de leitura minuciosa, crítica e reflexiva (figura 1).

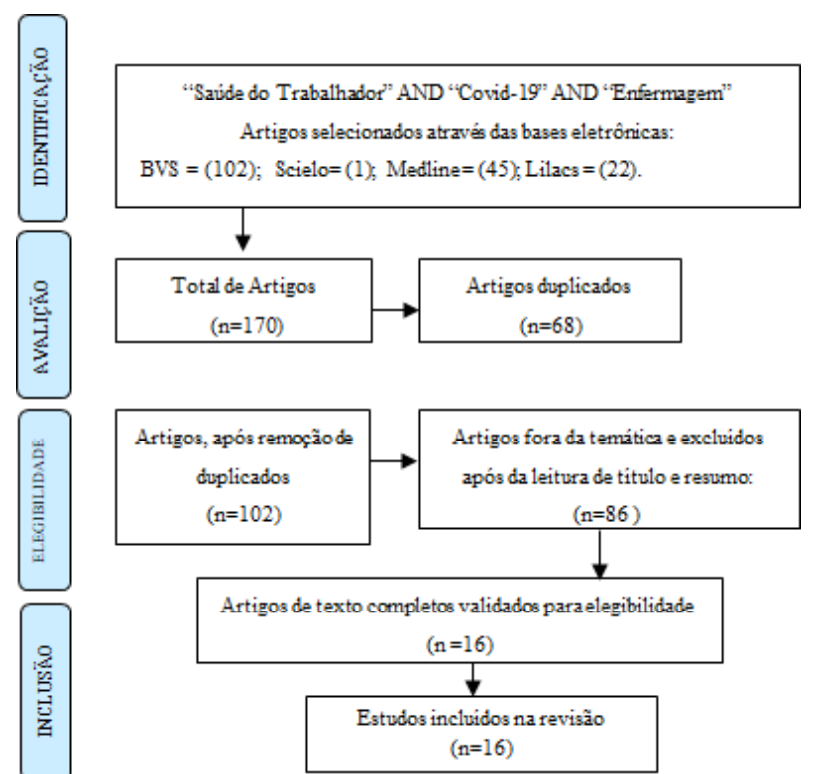

Figura 1. Fluxograma de artigos selecionados. Niterói, Rio de Janeiro, Brasil.
A partir disso, no quadro 1, encontram-se distribuídos os artigos selecionados e incluídos nesta revisão integrativa, sendo comparados por meio de análise crítica dos conteúdos apresentados.

A seleção foi composta por 16 artigos, como aponta o quadro 1. Quanto ao ano de publicação, constatou-se que, em sua totalidade, os artigos foram publicados no ano de 2020 mediante a atual emergência da pandemia. Em sua maioria (quatro) foram indexados nas bases Lilacs, Medline e Scielo.

Com relação ao idioma, oito artigos foram escritos em português $(50 \%)$ e sete artigos em inglês (40\%), e apenas um disponibilizado na língua espanhola (10\%), o que representa a predominância de estudos em português sobre o tema abordado.

Após análise textual e observação dos temas, emergiram as seguintes categorias: aspectos epidemiológicos relacionados à covid-19 e aspectos relacionados às condições de trabalho.

\section{Discussão}

\section{Aspectos epidemiológicos relacionados à covid-19}

Em decorrência da magnitude da doença conhecida como covid-19, diversos países enfrentam uma crise sanitária sem precedentes. A emergência da doença e a decorrente crise mundial ampliaram a demanda dos serviços de assistência e, consequentemente, a exposição dos profissionais de saúde à infecção pelo SARS-CoV-2 11 .

Enfermeiros representam mais da metade de todos os profissionais de saúde do mundo, fornecendo serviços vitais em todo o sistema de saúde. Historicamente, esses profissionais estão na vanguarda do combate a epidemias e pandemias que ameaçam a saúde. Em todo o mundo, estão demonstrando sua compaixão, bravura e coragem ao responder à pandemia de covid-19; nunca antes seu valor foi demonstrado com tanta clareza ${ }^{12}$.

O relatório, lançado pela Organização Mundial de Saúde (OMS) em parceria com o Conselho Internacional de Enfermeiras (ICN) e Nursing Now, revela que hoje existem pouco menos de 28 milhões de enfermeiros em todo o mundo. Mas isso ainda deixa um déficit global de 5,9 milhões de profissionais ${ }^{12}$. É revelador que mais de $80 \%$ das enfermeiras e enfermeiros do mundo trabalham em países que abrigam metade da população mundial ${ }^{12}$

São graves os impactos em termos de saúde pública frente ao vírus de fácil e rápida propagação na população, que leva à mudança abrupta nas rotinas dos serviços de saúde, como a intensificação de internações hospitalares por complicações respiratórias ${ }^{13}$. Além 
Quadro 1. Distribuição dos artigos incluídos na revisão. Niterói, Rio de Janeiro, Brasil, 2021.

\begin{tabular}{|c|c|c|c|}
\hline $\begin{array}{l}\text { PERIÓDICO/ } \\
\text { BASE }\end{array}$ & $\begin{array}{l}\text { TÍTULO/AUTORES/ } \\
\text { IDIOMA/ANO/PAÍS }\end{array}$ & $\begin{array}{l}\text { OBJETIVO/ } \\
\text { MÉTODO }\end{array}$ & PRINCIPAIS ACHADOS \\
\hline $\begin{array}{l}\text { Epidemiologia e } \\
\text { serviços de saúde: } \\
\text { revista do Sistema } \\
\text { Único de Saúde do } \\
\text { Brasil. } \\
\text { BVS. }\end{array}$ & $\begin{array}{l}\text { Description of covid-19 hospitali- } \\
\text { zed health worker cases in the first } \\
\text { nine weeks of the pandemic, Bra- } \\
\text { zil, } 2020 . \\
\text { Duarte MMS, Haslett MIC, Freitas } \\
\text { LJA, Gomes NTN et al. } \\
\text { Inglês/2020/ Brasil. }\end{array}$ & $\begin{array}{l}\text { Descrever os casos hospitalizados } \\
\text { pela covid-19 em profissionais de } \\
\text { saúde no Brasil. } \\
\text { Estudo descritivo de tipo série de } \\
\text { casos; foram incluídos aqueles com } \\
\text { adoecimento entre } 21 \text { de fevereiro e } \\
15 \text { de abril de } 2020 \text {, registrados no } \\
\text { Sistema de Informação de Vigilância } \\
\text { da Gripe (SIVEP-Gripe). }\end{array}$ & $\begin{array}{l}\text { O perfil dos trabalhadores de saúde hos- } \\
\text { pitalizados no covid-19 é semelhante ao } \\
\text { da população em geral no que diz res- } \\
\text { peito à idade e comorbidades, mas dife- } \\
\text { rente em relação ao sexo. As áreas mais } \\
\text { afetadas foram enfermagem e medicina. }\end{array}$ \\
\hline $\begin{array}{l}\text { Revista Enferma- } \\
\text { gem UERJ. }\end{array}$ & $\begin{array}{l}\text { Prevenção relacionada à exposição } \\
\text { ocupacional do profissional de saú- } \\
\text { de no cenário de covid-19. } \\
\text { Cristiane Helena Gallasch, Márcia } \\
\text { Lima da Cunha, Larissa Admá de } \\
\text { Souza Pereira, João Silvestre Sil- } \\
\text { va-Junior. } \\
\text { Português/2020/ Brasil. }\end{array}$ & $\begin{array}{l}\text { Descrever as principais recomenda- } \\
\text { ções sobre ações de prevenção de } \\
\text { contágio relacionadas à exposição } \\
\text { ocupacional dos profissionais de saú- } \\
\text { de atuantes frente à covid-19, dispo- } \\
\text { níveis até março de } 2020 \text {. } \\
\text { Artigo de atualidades. }\end{array}$ & $\begin{array}{l}\text { Os cuidados para prevenção de contami- } \\
\text { nação de trabalhadores nesta pandemia } \\
\text { pelo novo coronavírus devem ser prio- } \\
\text { rizados, evitando impactos negativos na } \\
\text { assistência à população que busca aten- } \\
\text { dimento nos serviços de saúde. }\end{array}$ \\
\hline Scielo Preprints. & $\begin{array}{l}\text { Context of nursing work in the co- } \\
\text { vid-19 pandemic and repercussions } \\
\text { for the mental health of workers. } \\
\text { Norma Valéria Dantas de Oliveira } \\
\text { Souza, Eloá Carneiro Carvalho, } \\
\text { Samira Silva Santos Soares et al. } \\
\text { Inglês/2020/Brasil. }\end{array}$ & $\begin{array}{l}\text { Refletir sobre o contexto de trabalho } \\
\text { dos profissionais de enfermagem na } \\
\text { pandemia da covid-19 e as repercus- } \\
\text { sões para saúde mental desses profis- } \\
\text { sionais. } \\
\text { Trata-se de um estudo teórico reflexi- } \\
\text { vo com dois eixos temáticos: } \\
\text { I) Pandemia pela covid-19: quadro } \\
\text { epidemiológico, manifestações e me- } \\
\text { didas preventivas; } \\
\text { II) Precarização laboral em tempos } \\
\text { de pandemia e impactos na saúde } \\
\text { mental do trabalhador. }\end{array}$ & $\begin{array}{l}\text { O presente artigo traz à tona a agudiza- } \\
\text { ção do cenário que eleva o potencial de } \\
\text { impacto negativo na saúde mental dos } \\
\text { trabalhadores de enfermagem. }\end{array}$ \\
\hline $\begin{array}{l}\text { Enferm. foco } \\
\text { (Brasília). } \\
\text { Lilacs. }\end{array}$ & $\begin{array}{l}\text { Profissional de saúde: segunda ví- } \\
\text { tima da pandemia covid-19. } \\
\text { Bohomol, Elena; Silva, Lúcia Mar- } \\
\text { ta Giunta da; Siqueira, Lucíola De- } \\
\text { mery et al. } \\
\text { Português/2020/Brasil. }\end{array}$ & $\begin{array}{l}\text { Refletir sobre a teoria ambientalista } \\
\text { de Florence Nightingale, contextuali- } \\
\text { zando a enfermagem moderna frente } \\
\text { suas origens e o desenvolvimento de } \\
\text { sua prática. } \\
\text { Estudo reflexivo, de perspectiva his- } \\
\text { tórica e que utiliza a pesquisa docu- } \\
\text { mental como método de investiga- } \\
\text { ção. Tomou por base especialmente } \\
\text { artigos de periódicos científicos e } \\
\text { livros clássicos sobre a história da } \\
\text { enfermagem e suas concepções, sem } \\
\text { limite de data de publicação. }\end{array}$ & $\begin{array}{l}\text { Compartilhar informações de outros } \\
\text { países e instituições que passam ou pas- } \\
\text { saram pela pandemia traz agilidade no } \\
\text { auxílio aos profissionais de saúde que } \\
\text { vivenciam experiências equivalentes. }\end{array}$ \\
\hline $\begin{array}{l}\text { Palliative Medicine. } \\
\text { Medline. }\end{array}$ & $\begin{array}{l}\text { Prioritising workforce wellbeing } \\
\text { and resilience: What covid-19 is } \\
\text { reminding us about self-care and } \\
\text { staff support. } \\
\text { Mills, Jason; Ramachenderan, Jo- } \\
\text { nathan; Chapman, Michael; Gre- } \\
\text { enland, Rohan; Agar, Meera. } \\
\text { Inglês/2020/Austrália. }\end{array}$ & Editorial. & $\begin{array}{l}\text { Embora a importância do autocuida- } \\
\text { do e do apoio da equipe possa parecer } \\
\text { evidente, até o momento seus papéis e } \\
\text { impacto sobre o bem-estar e a resiliência } \\
\text { na força de trabalho de cuidados paliati- } \\
\text { vos permanecem pouco pesquisados. }\end{array}$ \\
\hline
\end{tabular}


Quadro 1 (cont.). Distribuição dos artigos incluídos na revisão. Niterói, Rio de Janeiro, Brasil, 2021.

\begin{tabular}{|c|c|c|c|}
\hline $\begin{array}{l}\text { PERIÓDICO/ } \\
\text { BASE }\end{array}$ & $\begin{array}{l}\text { TÍTULO/AUTORES/ } \\
\text { IDIOMA/ANO/PAIIS }\end{array}$ & $\begin{array}{l}\text { OBJETIVO/ } \\
\text { MÉTODO }\end{array}$ & PRINCIPAIS ACHADOS \\
\hline $\begin{array}{l}\text { Revista Brasileira } \\
\text { de Enfermagem. } \\
\text { Medline. }\end{array}$ & $\begin{array}{l}\text { A saúde mental da enfermagem no } \\
\text { enfrentamento da covid-19 em um } \\
\text { hospital universitário regional. } \\
\text { Eduardo Bassani Dal'Bosco; } \\
\text { Lara Simone Messias Floriano; } \\
\text { Suellen Vienscoski Skupien; } \\
\text { Guilherme Arcaro; et al. } \\
\text { Português/2020/Brasil. }\end{array}$ & $\begin{array}{l}\text { Identificar a prevalência e fatores as- } \\
\text { sociados à ansiedade e depressão em } \\
\text { profissionais de enfermagem que atu- } \\
\text { am no enfrentamento da covid-19 em } \\
\text { hospital universitário. } \\
\text { Estudo observacional transversal, } \\
\text { com questionário sociodemográfico } \\
\text { e Escala de Medida de Ansiedade e } \\
\text { Depressão, com } 88 \text { profissionais de } \\
\text { enfermagem. Os dados foram anali- } \\
\text { sados por meio de frequência abso- } \\
\text { luta e relativa, utilizando o software } \\
\text { Statistical Package for the Social } \\
\text { Sciences. }\end{array}$ & $\begin{array}{l}\text { Foram evidenciados que, o processo de } \\
\text { trabalho da enfermagem, diante da pan- } \\
\text { demia da covid-19, possui impacto na } \\
\text { saúde mental dessa classe trabalhadora, } \\
\text { resultando em sinais de ansiedade e de- } \\
\text { pressão, indicando sofrimento psíquico } \\
\text { além daquele já intrínseco da profissão. } \\
\text { Assim se tornando essencial que esses } \\
\text { profissionais procurem apoio persona- } \\
\text { lizado, assim como capacitação para o } \\
\text { enfrentamento da pandemia. }\end{array}$ \\
\hline Medline. & $\begin{array}{l}\text { Covid-19: virtual occupational } \\
\text { skin health clinics for healthcare } \\
\text { workers. } \\
\text { Mahbub M Chowdhury; Nicola } \\
\text { Bevan; Karen Ryan. } \\
\text { Inglês/2020/Reino Unido. }\end{array}$ & Carta ao editor & $\begin{array}{l}\text { Foram examinados até o momento mais } \\
\text { de } 45 \text { profissionais de saúde e permiti- } \\
\text { do que os mesmos auto cuidassem de } \\
\text { seus problemas de pele relacionados ao } \\
\text { uso da máscara FFP3 ou qualquer outro } \\
\text { EPI utilizado pela enfermagem de saúde } \\
\text { ocupacional ou suporte médico. } \\
\text { Os dados sobre as lesões de pele encon- } \\
\text { tradas serão adicionados ao estudo mul- } \\
\text { ticêntrico junto à Sociedade Britânica de } \\
\text { Alergia Cutânea. }\end{array}$ \\
\hline $\begin{array}{l}\text { Infection Control \& } \\
\text { Hospital Epidemio- } \\
\text { logy. } \\
\text { Medline. }\end{array}$ & $\begin{array}{l}\text { Impact of anxiety and fear for } \\
\text { covid-19 toward infection control } \\
\text { practices among Thai healthcare } \\
\text { workers. } \\
\text { Anucha ApisarnthanaraK; Piya- } \\
\text { porn Apisarnthanarak; Chanida } \\
\text { Siripraparat; Pavarat Saengaram; } \\
\text { Narakorn Leeprechano; David J. } \\
\text { Weber. } \\
\text { Inglês/2020/Tailândia. }\end{array}$ & $\begin{array}{l}\text { Avaliar o impacto das emoções du- } \\
\text { rante a pandemia de covid-19 para as } \\
\text { práticas de prevenção de infecção em } \\
4 \text { hospitais. } \\
\text { Pesquisa realizada em } 2 \text { hospitais } \\
\text { universitários } \\
\text { (Hospital Universitário Thammasat, } \\
\text { Khlong Nueng, Pratum Thani, } \\
\text { Tailândia e Siriraj Hospital, Bangkok, } \\
\text { Tailândia) e } 2 \text { particulares hospitais } \\
\text { (Bumrungrad Hospital, Bangkok e } \\
\text { Rutnin Hospital, } \\
\text { Bangkok) de } 1^{\circ} \text { a } 31 \text { de março de } \\
2020 .\end{array}$ & $\begin{array}{l}\text { Apesar das limitações do estudo, foi } \\
\text { constatado que os profissionais da saú- } \\
\text { de que participaram da amostra, destes } \\
28 \% \text { eram profissionais de enfermagem, } \\
\text { demonstraram estar com medo e/ou so- } \\
\text { brecarregados, não estando dispostos a } \\
\text { receber ou ver novos pacientes inter- } \\
\text { nados com covid. Ressalta-se a impor- } \\
\text { tância de os hospitais terem políticas } \\
\text { de preparação, estoque adequado de } \\
\text { equipamentos de proteção e educação } \\
\text { continuada sobre transmissão, infeç̧ão } \\
\text { e prevenção de doenças. }\end{array}$ \\
\hline $\begin{array}{l}\text { REVENF - Portal } \\
\text { de Revistas de En- } \\
\text { fermagem. } \\
\text { BVS. }\end{array}$ & $\begin{array}{l}\text { Nursing Committee to Coping with } \\
\text { covid-19 in Bahia. } \\
\text { Tatiane Araújo dos Santos; Han- } \\
\text { derson Silva Santos; Mariana de } \\
\text { Almeida Moraes; Fernanda Car- } \\
\text { neiro Mussi. } \\
\text { Inglês/2020/Brasil. }\end{array}$ & $\begin{array}{l}\text { Relatar a experiência do Comitê de } \\
\text { Enfermagem para Enfrentamento da } \\
\text { covid-19 na Bahia. } \\
\text { O relato de experiência descreve mo- } \\
\text { tivação, objetivos, representantes, } \\
\text { organização, grupos de trabalhos, } \\
\text { atividades e impacto das ações do } \\
\text { Comitê. }\end{array}$ & $\begin{array}{l}\text { Os resultados dos trabalhos do Comitê } \\
\text { contribuem para orientar, apoiar, valori- } \\
\text { zar e defender os trabalhadores de enfer- } \\
\text { magem no enfrentamento do covid- } 19 \text {. }\end{array}$ \\
\hline $\begin{array}{l}\text { Revista Cubana de } \\
\text { Enfermaría. }\end{array}$ & $\begin{array}{l}\text { Reflexiones sobre los riesgos ocu- } \\
\text { pacionales en trabajadores de salud } \\
\text { en tiempos pandémicos por covid- } \\
19 . \\
\text { Joyce Soares e Silva; Ana Raquel } \\
\text { Batista de Carvalho; Hillda Dan- } \\
\text { dara Carvalho Santos Leite; Erika } \\
\text { Morganna Neves de Oliveira. } \\
\text { Espanhol/2020/Cuba. }\end{array}$ & $\begin{array}{l}\text { Refletir sobre os riscos ocupacionais } \\
\text { que envolvem os trabalhadores da } \\
\text { saúde na nova pandemia do corona- } \\
\text { vírus (covid-19). } \\
\text { Ensaio qualitativo teórico-reflexivo, } \\
\text { com base na literatura nacional e in- } \\
\text { ternacional. A busca dos estudos foi } \\
\text { possível devido à escolha dos descri- } \\
\text { tores controlados: "Riscos ocupacio- } \\
\text { nais", "Pessoal de saúde", "Infecções } \\
\text { por Coronavírus", "Coronavírus" e } \\
\text { extensas bases de dados latino-ame- } \\
\text { ricanas e internacionais. nos últimos } \\
\text { cinco anos. }\end{array}$ & $\begin{array}{l}\text { É fundamental conhecer e controlar os } \\
\text { riscos a que estão expostos os trabalha- } \\
\text { dores da saúde e, para tanto, estabelecer } \\
\text { estratégias de prevenção e minimização } \\
\text { das doenças. }\end{array}$ \\
\hline
\end{tabular}


Quadro 1 (cont.). Distribuição dos artigos incluídos na revisão. Niterói, Rio de Janeiro, Brasil, 2021.

\begin{tabular}{|c|c|c|c|}
\hline $\begin{array}{l}\text { PERIÓDICO/ } \\
\text { BASE }\end{array}$ & $\begin{array}{l}\text { TÍTULO/AUTORES/ } \\
\text { IDIOMA/ANO/PAÍS }\end{array}$ & $\begin{array}{l}\text { OBJETIVO/ } \\
\text { MÉTODO }\end{array}$ & PRINCIPAIS ACHADOS \\
\hline $\begin{array}{l}\text { Revista Brasileira de } \\
\text { Saúde Ocupacional. }\end{array}$ & $\begin{array}{l}\text { Saúde e segurança de profissionais } \\
\text { de saúde no atendimento a pacien- } \\
\text { tes no contexto da pandemia de } \\
\text { covid-19: revisão de literatura. } \\
\text { Adalgisa Peixoto Ribeiro, Gra- } \\
\text { ziella Lage Oliveira, Luiz Sergio } \\
\text { Silva, Edinilsa Ramos de Sousa. } \\
\text { Português/2020/Brasil. }\end{array}$ & $\begin{array}{l}\text { Analisar a produção científica sobre } \\
\text { a saúde dos trabalhadores da Saúde } \\
\text { que atendem pacientes no contexto } \\
\text { da pandemia de covid- } 19 \text {. } \\
\text { Realizou-se revisão de literatura que } \\
\text { incluiu artigos publicados em } 2020 \text {, } \\
\text { indexados nas bases PubMed, Web } \\
\text { of Science e na Biblioteca Virtual em } \\
\text { Saúde (Medline e Lilacs). }\end{array}$ & $\begin{array}{l}\text { Com a emergência sanitária em de- } \\
\text { corrência da pandemia causada pelo } \\
\text { SARS-Cov-2, importantes questões de } \\
\text { proteção e cuidados com a saúde dos } \\
\text { trabalhadores estão surgindo. A análise } \\
\text { das publicações mostrou que a ciência } \\
\text { tem cumprido seu papel e tem ofereci- } \\
\text { do à sociedade respostas rápidas, con- } \\
\text { siderando a gravidade e a velocidade } \\
\text { de disseminação da covid-19, pois um } \\
\text { número razoável de publicações sobre } \\
\text { o tema dos trabalhadores e sua saúde já } \\
\text { está disponível. }\end{array}$ \\
\hline Esc. Anna Nery. & $\begin{array}{l}\text { De cuidador a paciente: na pande- } \\
\text { mia da covid-19, quem defende e } \\
\text { cuida da enfermagem brasileira? } \\
\text { Soares, Samira Silva Santos et al. } \\
\text { Português/2020/Brasil. }\end{array}$ & $\begin{array}{l}\text { Refletir sobre a saúde do trabalhador } \\
\text { de enfermagem diante da crise da } \\
\text { pandemia pela covid-19. } \\
\text { Artigo de reflexão cujo tema envolve } \\
\text { a pandemia pela covid-19 e a saúde } \\
\text { do trabalhador de enfermagem. De- } \\
\text { senvolveu-se a partir de duas seções } \\
\text { teóricas: I) pandemia, aspectos epi- } \\
\text { demiológicos e recomendações para } \\
\text { proteção do trabalhador de enferma- } \\
\text { gem; II) saúde do trabalhador da en- } \\
\text { fermagem no contexto da covid-19. }\end{array}$ & $\begin{array}{l}\text { Os riscos à saúde do trabalhador de en- } \\
\text { fermagem, que já eram preocupantes an- } \\
\text { tes da pandemia, tornaram-se alarman- } \\
\text { tes no atual contexto, especialmente por } \\
\text { conta da incapacidade de um sistema } \\
\text { de saúde há muito precarizado. Tal fato } \\
\text { gera dilemas éticos, sofrimento físico e } \\
\text { psíquico aos trabalhadores de enferma- } \\
\text { gem, além de adoecimentos e mortes. }\end{array}$ \\
\hline $\begin{array}{l}\text { Comunicação em } \\
\text { Ciências da Saúde. }\end{array}$ & $\begin{array}{l}\text { Fatores de estresse nos profissio- } \\
\text { nais de enfermagem no combate à } \\
\text { pandemia da covid-19: Síntese de } \\
\text { Evidências. } \\
\text { Diogo Jacintho Barbosa; Márcia } \\
\text { Pereira Gomes; Fabiana Barbosa } \\
\text { Assumpção de Souza; Antônio } \\
\text { Marcos Tosoli Gomes. } \\
\text { Português/2020/Brasil. }\end{array}$ & $\begin{array}{l}\text { Identificar os principais efeitos psico- } \\
\text { lógicos da pandemia da covid-19 nos } \\
\text { profissionais de enfermagem; descre- } \\
\text { ver os principais fatores capazes de } \\
\text { gerar estresse psicológico nos profis- } \\
\text { sionais de enfermagem; descrever as } \\
\text { estratégias de coping para o combate } \\
\text { ao estresse emocional. } \\
\text { Revisão da literatura, utilizando-se } 5 \\
\text { artigos indexados no PubMed e Li- } \\
\text { lacs }\end{array}$ & $\begin{array}{l}\text { O aumento da carga de trabalho, medo } \\
\text { de contaminar os familiares e de } \\
\text { se contaminar, desinformação e raiva } \\
\text { do governo e dos sistemas de saúde são } \\
\text { os principais fatores capazes de gerar } \\
\text { estresse emocional nos profissionais de } \\
\text { enfermagem. }\end{array}$ \\
\hline $\begin{array}{l}\text { Cogitare enferma- } \\
\text { gem. } \\
\text { Lilacs. }\end{array}$ & $\begin{array}{l}\text { Condições de trabalho e o impacto } \\
\text { na saúde dos profissionais de en- } \\
\text { fermagem frente a covid-19. } \\
\text { Fernanda Moura D'Almeida Mi- } \\
\text { randa, Leni de Lima Santana, Ali- } \\
\text { ne Cecília Pizzolato, Leila Maria } \\
\text { Mansano Saquis. } \\
\text { Português/2020/Brasil. }\end{array}$ & $\begin{array}{l}\text { Refletir sobre as condições de traba- } \\
\text { lho dos profissionais de enfermagem } \\
\text { no enfrentamento ao novo coronaví- } \\
\text { rus e apontar o impacto na vida desses } \\
\text { profissionais em meio à pandemia. } \\
\text { Artigo de reflexão. }\end{array}$ & $\begin{array}{l}\text { As fragilidades encontradas no cotidiano } \\
\text { laboral dos profissionais de enfermagem } \\
\text { são descritas pela literatura nacional e } \\
\text { internacional, nas quais estão incluídas } \\
\text { as más condições de trabalho, sobrecar- } \\
\text { ga física e mental, baixa remuneração e } \\
\text { ausência de Equipamentos de Proteção } \\
\text { Individual adequados para o enfrenta- } \\
\text { mento desse agravo. }\end{array}$ \\
\hline $\begin{array}{l}\text { Revista Latino- } \\
\text { Americana de En- } \\
\text { fermagem. } \\
\text { Scielo. }\end{array}$ & $\begin{array}{l}\text { Desafios de profissionais de Enfer- } \\
\text { magem Pediátrica frente à pande- } \\
\text { mia da covid-19. } \\
\text { Góes FGB, Silva ACSS, Santos } \\
\text { AST, Pereira-Ávila FMV, Silva LJ, } \\
\text { Silva LF, Goulart MCL. } \\
\text { Português/2020/Brasil. }\end{array}$ & $\begin{array}{l}\text { Identificar os desafios de profissio- } \\
\text { nais de Enfermagem Pediátrica frente } \\
\text { à pandemia da covid-19. } \\
\text { Pesquisa qualitativa, desenvolvida } \\
\text { por meio de formulário eletrônico } \\
\text { semiestruturado entre profissionais } \\
\text { de Enfermagem que atuam em unida- } \\
\text { des pediátricas no Estado do Rio de } \\
\text { Janeiro. }\end{array}$ & $\begin{array}{l}\text { Distintos desafios referentes à pandemia } \\
\text { da covid-19 foram relatados, dentre eles, } \\
\text { a promoção da assistência integral e de } \\
\text { qualidade frente à preocupação quanto à } \\
\text { proteção de si e do outro, com destaque } \\
\text { para o sentimento de medo. A falta de } \\
\text { equipamentos de proteção individual, } \\
\text { treinamentos, testes diagnósticos e co- } \\
\text { nhecimentos/informações relacionados } \\
\text { à doença, o número reduzido de profis- } \\
\text { sionais de Enfermagem e a desvaloriza- } \\
\text { ção da categoria também foram sinali- } \\
\text { zados. }\end{array}$ \\
\hline
\end{tabular}


Quadro 1 (cont.). Distribuição dos artigos incluídos na revisão. Niterói, Rio de Janeiro, Brasil, 2021.

\begin{tabular}{|c|c|c|c|}
\hline $\begin{array}{c}\text { PERIÓDICO/ } \\
\text { BASE }\end{array}$ & $\begin{array}{l}\text { TÍTULO/AUTORES/ } \\
\text { IDIOMA/ANO/PAÍS }\end{array}$ & $\begin{array}{l}\text { OBJETIVO/ } \\
\text { MÉTODO }\end{array}$ & PRINCIPAIS ACHADOS \\
\hline $\begin{array}{l}\text { GMS- Germany } \\
\text { Medical Science. } \\
\text { NCBI. }\end{array}$ & $\begin{array}{l}\text { Psychosocial burden of healthcare } \\
\text { professionals in times of covid-19 } \\
\text { - a survey conducted at the Uni- } \\
\text { versity Hospital Augsburg. } \\
\text { Zerbini, et al. } \\
\text { Inglês/2020/Alemanha. }\end{array}$ & $\begin{array}{l}\text { Investigar a carga psicossocial de } \\
\text { médicos e enfermeiras dependendo } \\
\text { do seu grau de contato com pacientes } \\
\text { covid-19. } \\
\text { Os participantes preencheram dois } \\
\text { questionários padronizados (o Pa- } \\
\text { tient Health Questionnaire, PHQ; e o } \\
\text { Maslach Burnout Inventory, MBI) e } \\
\text { relataram seu medo da infecção por } \\
\text { covid-19 e estresse no trabalho pela } \\
\text { escala Likert de } 10 \text { pontos. Finalmen- } \\
\text { te, eles responderam a três perguntas } \\
\text { abertas sobre as causas da carga, re- } \\
\text { cursos de apoio e necessidades du- } \\
\text { rante a crise. }\end{array}$ & $\begin{array}{l}\text { Enfermeiros que trabalham nas enfer- } \\
\text { marias do covid-19 relataram níveis } \\
\text { mais elevados de estresse, exaustão e } \\
\text { humor depressivo, bem como níveis } \\
\text { mais baixos de realização relacionada ao } \\
\text { trabalho em comparação com seus cole- } \\
\text { gas nas enfermarias regulares. Os médi- } \\
\text { cos relataram pontuações semelhantes, } \\
\text { independentemente de seu contato com } \\
\text { pacientes covid-19. As causas mais co- } \\
\text { muns de sobrecarga foram estresse no } \\
\text { trabalho e incerteza sobre o futuro. }\end{array}$ \\
\hline
\end{tabular}

disso, a situação atual requer uma robusta estrutura hospitalar que possibilite tomada de decisões rápidas e adequadas para o controle da propagação do vírus, incluindo-se rede integrada aos sistemas de Saúde Pública a qual permita melhor enfrentamento e atuação dos profissionais envolvidos situação ${ }^{14}$.

O espectro clínico da infecção por covid-19 varia de infecção assintomática ou gripe leve até condições respiratórias mais graves, como a síndrome respiratória aguda grave (SARS). O Brasil possui sistema de vigilância para infecções por vírus respiratórios que visa identificar, registrar e monitorar o quadro clínico e epidemiológico, bem como as características desses casos ${ }^{15}$. A covid-19 é de notificação compulsória imediata pelos serviços públicos e privados, e os casos suspeitos e/ou confirmados devem ser registrados no sistema oficial do Ministério da Saúde ${ }^{16}$.

No enfrentamento da pandemia, destaca-se o papel da enfermagem nas ações de vigilância, prevenção, controle da transmissão do vírus, assistência aos enfermos, pesquisas sobre a covid-19 e nas orientações à comunidade. Reforça-se o olhar atento da profissão ao cuidado do ser humano, do ambiente, da família e coletividade, com empatia e acolhimento ${ }^{17}$.

Por fim, é necessário que se estabeleça o perfil dos profissionais de saúde hospitalizados pela infecção do novo coronavírus no Brasil, para direcionar as ações de vigilância epidemiológica. Identificou-se que os casos de covid-19 ocorriam, predominantemente, entre o sexo feminino, adultos jovens, profissionais da medicina e da enfermagem, que apresentaram febre, tosse, dispneia, cardiopatia, diabetes mellitus e asma mais frequentemente ${ }^{18}$.

\section{Aspectos relacionados às condições de trabalho}

O enfrentamento da pandemia da covid- 19 está tornando mais evidente as demandas históricas da enfermagem quanto às condições de trabalho, à extensão da jornada laboral, ao dimensionamento de pessoal, à remuneração e à visibilidade social da categoria ${ }^{19}$.

Sabe-se que o enfrentamento da doença expõe os profissionais a diversas situações de risco laboral, com destaque para a exposição repetida ao patógeno; extensas jornadas de trabalho, associadas a múltiplos vínculos; cansaço; estresse ocupacional; estigmatização; violências física e psicológica ${ }^{18}$.

Dados divulgados pela OMS revelam que cerca de 570 mil profissionais de saúde foram infectados e 2,5 mil morreram devido às complicações da covid-19, nas Américas no ano de 2020. Neste mesmo ano, os Estados Unidos da América e o México têm as contagens de casos mais altas do mundo ${ }^{20}$.

Paralelo a isso, no contexto mundial, a literatura descreve que em países como Portugal, Alemanha e Austrália, o enfrentamento da escassez de enfermeiros nos hospitais impõe uma sobrecarga maior diante do cenário pandêmico, o que reflete diretamente na saúde do profissional e do paciente. No Brasil, apesar da inexistência de dados oficiais do Ministério da Saúde referentes ao adoecimento dos profissionais de enfermagem, o Conselho Federal de Enfermagem, por meio do Observatório da Enfermagem, confirmou 143 mortes de profissionais e 17.044 casos de infectados ${ }^{21}$.

A sobrecarga no atendimento dos pacientes vitimados pela doença gera certa situação extrema e estressante para os profissionais relacionada à múltiplos fatores como alarme social, falta de recursos, saturação dos serviços e incerteza, transformando-o na segunda vítima desse fenômeno ${ }^{22}$.

Além das situações apresentadas anteriormente, a pandemia imprimiu impacto considerável no adoecimento mental, tornando os profissionais da saúde, particularmente os de enfermagem, mais expostos 
e vulneráveis ao adoecimento mental e sofrimento psíquico, devido às vivências decorrentes da doença ${ }^{17}$.

Asíndrome de burnout é muito comum em pessoas que permanecem em locais de trabalho por longas horas, além de possuírem grande carga de trabalho. A doença pode ser compreendida como o esgotamento profissional decorrente da tensão emocional crônica vivida pelo trabalhador, associada à despersonalização e baixa realização pessoal ${ }^{23}$. Essa doença pode ser realidade em ambientes de saúde atuais, visto que a situação impõe o risco de adoecimento ou infecção elevados, excesso de trabalho em situação adversa e insegurança para realização de procedimentos diante da crise $^{24}$.

$\mathrm{Na}$ China, mais precisamente na província de Wuhan em Hubei, onde o surto começou, foram identificados problemas psicológicos, incluindo ansiedade, depressão e estresse entre os profissionais de saúde ${ }^{25}$. Há evidências de que o estresse psicológico e a ansiedade podem ser alguns dos fatores de risco para maior morbidade mediada pela covid-19 entre a equipe de saúde ${ }^{8}$.

Ademais, dos riscos habituais envolvidos no exercício das profissões da área da saúde, há também a problemática da disponibilidade de materiais de proteção adequados e em quantidades suficientes, constituindo outro desafio imposto pela pandemia atual ${ }^{18}$.

A legislação trabalhista brasileira, via Norma Regulamentadora de Segurança e Saúde no Trabalho em Serviços de Saúde (NR32), indica a obrigatoriedade do empregador fornecer ao trabalhador EPI em quantidade suficiente, descartáveis ou não, para o desenvolvimento seguro das tarefas de trabalho. Além do fornecimento, deve-se assegurar a capacitação de forma contínua e a garantia de proteção ao trabalhador, sempre que houver mudança das condições de exposição a agentes biológicos ${ }^{26}$.

As constantes situações de morte e estresse vivenciados em ambientes, muitas vezes, sobrecarregados de pacientes com alto poder de transmissibilidade viral, requerem atendimento de enfermagem preciso e cauteloso, tanto nos procedimentos técnicos quanto na paramentação $\mathrm{e}$ desparamentação rigorosa ${ }^{27}$. Logo, os trabalhadores de enfermagem enfrentam importantes dilemas, como os que envolvem o atendimento de pacientes na ausência de condições e materiais adequados ${ }^{17}$.

Durante essa pandemia, notou-se a exacerbação do aparecimento de lesões e erosões na pele dos trabalhadores, devido a manipulação incorreta das máscaras de proteção e o seu uso prolongado. Dessa forma, demonstra-se importante discutir acerca dos materiais utilizados, bem como sua correta manipulação ${ }^{28}$.

Diante disso, mostra-se fundamental que os profissionais de enfermagem se preparem para enfrentar a ameaça viral, assumindo postura resiliente frente ao desafio, buscando informações e estratégias de enfrentamento, como apoio psicológico especializado, atendimento por telefone que realize escuta diferenciada, sigilosa e gratuita, realização de práticas integrativas complementares (como Yoga e Reiki) e exercícios de relaxamento ${ }^{29}$.

Essas ações se fazem necessárias devido aos danos importantes à saúde mental dos profissionais da saúde e seus familiares, evidenciada nas obras analisadas. Em meio a esta crise sanitária, é preciso cuidar da saúde de quem cuida. Nesse sentido, é necessário fortalecer o trabalho em equipe multiprofissional, promover a comunicação eficaz entre as várias instâncias do trabalho em saúde, bem como intra e inter equipes ${ }^{30,31}$.

\section{Considerações finais}

A partir da literatura selecionada, pode-se perceber que dada a natureza recente da temática muitos estudos que contemplam o tema ainda estão sendo desenvolvidos e publicados. Espera-se que a produção científica sobre a saúde dos trabalhadores de enfermagem seja fomentada durante a pandemia, pois sua contribuição é de suma importância para o desenvolvimento de estratégias de prevenção de doenças e gerenciamento do estresse laboral, revelando-se importantes componentes do enfrentamento dos profissionais a situações delicadas como a pandemia do novo coronavírus.

A situação da saúde pública atual gerou diversas problemáticas recentes, incluindo o impacto negativo sobre a saúde mental dos trabalhadores, além de reverberar problemas conhecidos, que agora demonstram-se acentuados, como é o caso da escassez de equipamentos de proteção individual que os torna mais suscetíveis ao adoecimento.

Além de problemas estruturais, quantitativo de materiais e dimensionamento de pessoal, percebe-se que o estado emocional dos profissionais encontra fragilidade mediante à pandemia, onde imperam sentimentos de medo, insegurança e o adoecimento mental, no que tange a saúde do trabalhador de enfermagem.

Logo, é importante que o tema seja mais discutido, visto que o ano de 2020 foi celebrado como o ano da categoria profissional e pouco se fala dos danos causados a mesma no período pandêmico. Sabe-se que os profissionais são afetados diariamente com as consequências da doença e que precisam serconsiderados nessas discussões abrindo espaço para dividir suas histórias, aflições e medos. O suporte adequado deve ser fornecido pelas instituições, incluindo-se os profissionais afastados para que o cuidado também seja abrangente a todos aqueles que cuidam. 


\section{Referências}

1. WHO, World Health Organization. Coronavirus disease (COVID-19) Pandemic [Internet]. 2020 [acesso 12 Mar. 2021]. Disponível em: https:// www.who.int/emergencies/diseases/novel-coronavirus-2019.

OMS, Organização Pan-Americana da Saúde. Organização Mundial da Saúde [Internet]. 2021 [acesso 12 Mar. 2021]. Disponível em: https:// www.paho.org/pt/covid19.

Tavares V. A saúde dos que estão na linha de frente. Escola Politécnica de Saúde Joaquim Venâncio. EPSJV/Fiocruz. [Internet]. 2020 [acesso 17 abr. 2020]. Disponível em: http://www.epsjv.fiocruz.br/noticias/reportagem/asaude-dos-que-estao-na-linha-de-frente.

4. Ribeiro CJN, Santos AD, Lima SVMA, Ribeiro MCO. Intervenções de restrição de mobilidade social durante a pandemia da covid-19 e suas repercussões psicossociais no Brasil. Enferm. Foco [Internet]. 2020 [acesso 17 abr. 2020]; 11(1) Especial: 179-181. Disponível em: http://revista.cofen. gov.br/index.php/enfermagem/article/view/3412.

5. OMS, Organização Mundial da Saúde. OMS define 2020 como o ano internacional dos profissionais de enfermagem e obstetrícia [Internet]. Janeiro, 2020 [acesso 12 Mar. 2021]. Disponível em: https://bvsms.saude. gov.br/ultimas-noticias/3119-oms-define-2020-como-ano-internacionaldos-profissionais-de-enfermagem-e-obstetricia\#: :text=Segundo $\% 20 \mathrm{a} \% 20$ OMS $\% 2$ C $\% 20$ o $\% 20$ mundo,pessoal $\% 20$ de $\% 20$ enfermagem $\% 20$ e $\% 20$ obstetr $\% \mathrm{C} 3 \% \mathrm{ADcia}$.

6. Santos GBM, Lima RCD, Barbosa JPM, Silva MC, Andrade MAC Cuidado de si: trabalhadoras da saúde em tempos de pandemia pela Covid19. Trabalho, Educação e Saúde [Internet]; 2020 [acesso 17 abr. 2020] 18(3), e00300132. Disponível em: https://www.scielo.br/scielo.php?script=sci arttext\&pid=S1981-77462020000300518\&lng=en\&nrm=iso

7. Gomes CM, Vasconcellos LCF, Machado JMH. Saúde do trabalhador: aspectos históricos, avanços e desafios no Sistema Único de Saúde. Ciência \& Saúde Coletiva [Internet]. 2018 [acesso 02 Mar. 2021]; v. 23, n.6, pp. 1963-1970. Disponível em: https://doi.org/10.1590/141381232018236.04922018 .

8. Bezerra G, Sena AS, Braga S, dos Santos ME, Correia LF, Clementino KM, Carneiro YV, Pinheiro W. O impacto da pandemia por COVID-19 na saúde mental dos profissionais de saúde: revisão integrativa. REAID [Internet]. 4 set. 2020 [acesso 12 Mar. 2021]; 93:e-20012. Disponível em: http://www.revistaenfermagematual.com.br/index.php/revista/article/ view/758.

9. INS, Instituto Nacional de Salud. Tabla distribución de contagio por profesión. Consultado el 21 de julio de 2020 [Internet]. 2020 [acceso 12 Mar. 2021]. Disponible en: https://www.ins.gov.co/Noticias/Paginas/ Coronavirus.aspx.

10. Souza MT, Silva MD, Carvalho R. Revisão integrativa: o que é e como fazer. Einstein (São Paulo) [Internet]. 2010 Mar [acesso 12 Mar. 2021]; 8(1): 102-106. Disponível em: http://www.scielo.br/scielo.php?script=sci arttext\&pid=S1679-45082010000100102\&lng=en.

11. Lopez FG, Palotti PLDM, Barbosa SCT, Koga NM. Mapeamento dos profissionais de saúde no Brasil: alguns apontamentos em vista da crise sanitária da COVID-19. Brasília: Instituto de Pesquisa Econômica Aplicada [Internet]; 2020 [acesso 19 abr. 2020]. 13p. Disponível em: http://repositorio. ipea.gov.br/handle/11058/9837.

12. OMS, Organização Pan-Americana da Saúde Brasil. Dia Mundial da Saúde: OMS e parceiros pedem investimentos urgentes em profissionais de enfermagem [Internet]. Abril, 2020 [acesso 12 Mar. 2021]. Disponível em: https:/www.paho.org/bra/index. php?option $=$ com content $\&$ view $=$ article $\&$ id $=6134$ :dia-mundial-da-saudeoms-e-parceiros-pedem-investimentos-urgentes-em-profissionais-deenfermagem $\&$ Itemid $=844$

13. Gallasch CH, Cunha ML, Pereira LAS, Silva-Junior JS. Prevenção relacionada à exposição ocupacional: COVID-19. Revista Enfermagem UERJ [Internet]. 2020 [acesso 17 abr. 2020]; 28:e49596. Disponível em: https://www.e-publicacoes.uerj.br/index.php/enfermagemuerj/article/ view/49596.

14. Liu T, Hu J, Kang M, Lin L, Zhong H, Xiao J, et al. Transmission dynamics of 2019 novel coronavirus (2019-nCoV). Lancet [Internet]. Jan. 2020 [acesso 26 Mar. 2020]. Disponível em: https://www.biorxiv.org/conten $\mathrm{t} / 10.1101 / 2020.01 .25 .919787 \mathrm{v} 1$

15. Brasil, Ministério da Saúde (BR). Secretaria de Vigilância em Saúde. Guia de vigilância epidemiológica: emergência de saúde pública de importância nacional pela doença pelo coronavírus 2019 [Internet] Brasília: Ministério da Saúde; 2020 [acesso 20 abr. 2020]. Disponível em: https://portaldeboaspraticas.iff.fiocruz.br/biblioteca/guia-de-vigilanciaepidemiologica-emergencia-de-saude-publica-de-importancia-nacional/.

16. ANS, Agência Nacional de Saúde. Nota Informativa da ANS Número 1: Sobre Coronavírus - COVID-19 [Internet]. 2020 [acesso 20 Mar. 2020]. Disponível em: http://www.ans.gov.br/images/comunicado01 coronavirus.pdf.

17. Miranda FMD, Santana LL, Pizzolato AC, Saquis LMM. Condições de trabalho e o impacto na saúde dos profissionais de enfermagem frente a Covid-19. Cogitare enferm. [Internet] 2020 [acesso 20 Mar. 2020]; 25: e72702. Disponível em: https://revistas.ufpr.br/cogitare/article/view/72702/ pdf.

18. Duarte MMS, Freitas LJA, Haslett MIC, Gomes NTN, Malta JMAS Alves AJS et al. Descrição dos casos hospitalizados pela COVID-19 em profissionais de saúde nas primeiras nove semanas da pandemia, Brasil, 2020. Epidemiol. Serv. Saúde [Internet]. Set. 2020 [acesso 12 Mar. 2021]; 29 (5) 28. Disponível em: https://www.scielosp.org/article/ress/2020.v29n5/ e2020277/\#

19. COFEN, Conselho Federal de Enfermagem (BR). Demandas de décadas da Enfermagem se sobressaem no combate à pandemia [Internet]. Brasília, DF; 2020 [acesso 27 abr. 2020]. Disponível em: http://www.cofen. gov.br/demandas-de-decadas-da-enfermagem-se-sobressaem-no-combate-apandemia_78927.html.

20. OMS, Organização Pan-Americana da Saúde. Organização Mundial da Saúde. Cerca de 570 mil profissionais de saúde se infectaram e 2,5 mil morreram por COVID-19 nas Américas [Internet]. Set. 2020 [acesso 12 Mar. 2021]. Disponível em: https://www.paho.org/bra/index. php?option $=$ com content $\&$ view $=$ article $\&$ id $=6270$ : cerca-de-570-milprofissionais-de-saude-se-infectaram-e-2-5-mil-morreram-por-covid-19nas-americas\&Itemid=812\#: : text $=022$ Nossos $\% 20$ dados $\% 20$ mostram $\% 20$ que $\% 20$ quase,desta $\% 20$ pandemia $\% 20$ n $\%$ C 3\%A3o\%20tem $\% 20$ precedentes.

21. ANS, Agência Nacional de Vigilância Sanitária (BR). Nota Técnica GVIMS/GGTES/ANVISA No 04/2020: Orientações para serviços de saúde: medidas de prevenção e controle que devem ser adotadas durante a assistência aos casos suspeitos ou confirmados de infecção pelo novo coronavírus (SARS-COV-2) [Internet]. Brasília, DF: ANVISA; 2020 [acesso 22 abr. 2020]. Disponível em: https://www.gov.br/anvisa/pt-br/centraisdeconteudo/ publicacoes/servicosdesaude/notas-tecnicas/nota-tecnica-n-04-2020-gvimsggtes-anvisa-atualizada.pdf/view.

22. Bohomol E, Silva LMG, Siqueira LD, Velhote MCP, Fogliano RRF. Profissional de saúde: segunda vítima da pandemia covid-19. Enferm. Foco [Internet]. 2020 [acesso 12 Mar. 2021]; 11 (1) Especial: 84-91. Disponível em: http://biblioteca.cofen.gov.br/wp-content/uploads/2020/08/ ProfissionalSaudeCovid19.pdf.

23. Silva SCPS, Nunes MAP, Santana VR, Reis FP, Machado NJ, Lima SO. A síndrome de Burnout em profissionais da Rede de Atenção Primária à Saúde de Aracaju, Brasil. Ciênc. saúde coletiva [Internet]. 2015 [acesso 12 Mar. 2021]; 20(10): 3011-3020. Disponível em: http://www.scielo.br/scielo. php?script $=$ sci arttext\&pid=S1413-81232015001003011\&lng=en.

24. Polakiewicz R. Saúde mental de profissionais de enfermagem na pandemia de coronavírus. Saúde mental de profissionais de enfermagem na pandemia de coronavírus. Portal PEBMED [Internet]. 2020 [acesso 12 Mar. 2021]. Disponível em: https://pebmed.com.br/saude-mental-de-profissionaisde-enfermagem-na-pandemia-de-coronavirus/.

25. Yang Y, Li W, Zhang Q, Zhang L, Cheung T, Xiang Y. Mental health services for older adults in China during the COVID-19 outbreak. The Lancet Psychiatry [Internet]. 2020 [acesso 12 Mar. 2021];7(4):e19. Disponível em: https://www.thelancet.com/journals/lanpsy/article/PIIS22150366(20)30079-1/fulltext.

26. Brasil, Enit. Norma regulamentadora 32 (NR 32). Segurança e saúde no trabalho em serviços de saúde. Estabelecer diretrizes básicas para a implementação de medidas de proteção à segurança e à saúde dos trabalhadores dos serviços de saúde [Internet]. 06 de dezembro de 2002 [acesso 12 Mar. 2021]. Disponível em: http://www.guiatrabalhista.com.br/ legislacao/nr/nr32.htm 
27. Jackson D, Bradburry-Jones C, Baptiste D, Gelling L, Morin K, Neville S, et al. Life in the pandemic: some reflections on nursing in the context of COVID-19. J Clin Nurs. [Internet]. 2020 [acesso em 15 abr 2020]; Disponível em: http://doi.org/10.1111/jocn.15257.

28. Chowdhury MM, Bevan N, Ryan K. Covid-19: virtual occupational skin health clinics for healthcare workers. BMJ [Internet]. 2020 [acesso 12 Mar. 2021]; m2281. Disponível em: https://www.bmj.com/ content $/ 369 / \mathrm{bmj} . \mathrm{m} 2281 / \mathrm{rapid}-$ responses?utm_content=americas\&utm campaign=usage\&utm medium $=$ cpc\&utm source $=$ trendmd

29. Dal'bosco EB, Messias FLS, Vienscoski SS, Arcaro G, Martins AR, Anselmo ACC. A saúde mental da enfermagem no enfrentamento da COVID-19 em um hospital universitário regional. Rev. Bras. Enferm. [Internet]. 2020 [acesso 12 Mar. 2021]; e20200434. . Disponível em http://www.scielo.br/scielo.php?script=sci arttext\&pid=S0034$71672020001400153 \& \operatorname{lng}=$ pt\&nrm=iso.

30. United Nations (US). Policy brief: COVID-19 and the need for action on mental health [Internet]. New York: United Nations; 2020 [acesso 22 abr. 2020]. Disponível em: https://www.un.org/sites/un2.un.org/files/un_policy brief-covid_and_mental_health_final.pdf.

31. Pennafort VPS, Freitas CHA, Jorge MSB, Queiroz MVO, Aguiar CAA. Práticas integrativas e o empoderamento da enfermagem. Rev Min Enferm. [Internet]. 2012 [acesso 12 Mar. 2021]; 16(2):289-95. Disponível em: https://cdn.publisher.gn1.link/reme.org.br/pdf/v16n2a19.pdf. 